\title{
Altering critical depinning current via domain wall pile-up in magnetic nanowires
}

\author{
Liwei D. Geng and Yongmei M. Jin* \\ Materials Science and Engineering Department, Michigan Technological University, \\ Houghton, MI 49931, USA
}

\begin{abstract}
An important role of domain wall pile-up in current-driven domain wall depinning in magnetic nanowires is revealed using micromagnetic simulations. It is found that the critical current for domain wall depinning can be substantially reduced and conveniently tuned by controlling domain wall number in the pile-up at pinning site, in analogy to dislocation pile-up responsible for Hall-Petch effect in mechanical strength. Domain wall pinning and depinning at an s-shape bend is considered, and the effects of curvature and current crowding in magnetic circuit on domain wall behaviors are discussed.
\end{abstract}

\section{Introduction}

Domain walls in magnetic nanowires attract great attentions for their applications in advanced logic and memory devices [1,2]. Manipulation of domain walls by electric current is an essential component of domain wall devices and is under intensive study $[3,4]$. Pinning and depinning of domain walls is a critical issue for domain wall manipulation where pinning sites are usually turning corners, bends, joints, and notches in magnetic circuits [5,6]. Reducing the critical depinning current is important for technological application, in order to lower the power and to avoid increased nanowire temperature hindering reliable domain wall manipulation [7]. Finding an effective means to reduce and tune the critical depinning current will have a significant impact on the operation of domain wall devices. In this paper we present a micromagnetic simulation study of current-driven domain wall behaviors in thin film-patterned planar magnetic nanowires, which demonstrates a new mechanism for substantial reduction and effective tuning of the critical current for depinning domain walls in magnetic circuit. It is worth noting that micromagnetic simulation has been widely used to understand various factors that affect the critical current for domain wall depinning in magnetic nanowires, including edge roughness [8,9], thermal perturbation [10], magnetic anisotropy $[11,12]$ and saturation magnetization [13], applied magnetic field [14], and notch shape [15]. The new mechanism revealed in this work is based on domain wall pile-up, in analogy to the well-known dislocation pile-up mechanism responsible for the Hall-Petch effect in mechanical strength.

Since the pinning strength of a pinning site depends on its geometrical shape, the depinning current can be modified by changing the geometrical shape of the pinning site. For example, decreasing the curvature of a bend would reduce its pinning strength [6] and thus lower the critical depinning current. However, changing geometrical shape is constrained by other functional requirements of the pinning site being a part of the magnetic circuit, thus the changeable range of the critical depinning current is limited.

* Email: ymjin@mtu.edu 
Here we show that utilizing domain wall pile-up enables not only a drastic reduction in the critical depinning current but also an effective programing of the current-driven pinning and depinning operations of domain walls. As shown in Fig. 1(a), a domain wall pile-up is formed in magnetic nanowire when a set of $180^{\circ}$ and/or $360^{\circ}$ domain walls of the same chirality are pushed against a barrier (an s-shape pinning site here). For convenience of discussion, such a domain wall pile-up will be named after its total magnetization rotation angle, $n \pi$-pileup, where $n$ is an integer greater than $2(n=1$ and 2 correspond respectively to $180^{\circ}$ and $360^{\circ}$ domain wall). A $360^{\circ}$ domain wall is highly stable [16] unless an antiparallel magnetic field is applied, under which it dissociates into two $180^{\circ}$ domain walls. Unlike stable $180^{\circ}$ domain wall and relatively stable $360^{\circ}$ domain wall [17], an $n \pi$-pileup is not stable under magnetic field, dissociating into a set of discrete $360^{\circ}$ and $180^{\circ}$ domain walls, as exemplified in Fig. 1(b). However, an $n \pi$ pileup under spin-polarized current does not dissociate, but instead moves as a whole in the nanowire, as demonstrated in Fig. 1(c). When the current-driven motion of an $n \pi$ pileup encounters a barrier in its moving direction, interesting domain wall pinning and depinning phenomena take place, as will be discussed in the following. It is worth noting that a set of $180^{\circ}$ and/or $360^{\circ}$ domain walls of the same chirality (needed for formation of domain wall pile-up) can be conveniently generated in magnetic nanowires [18-20].

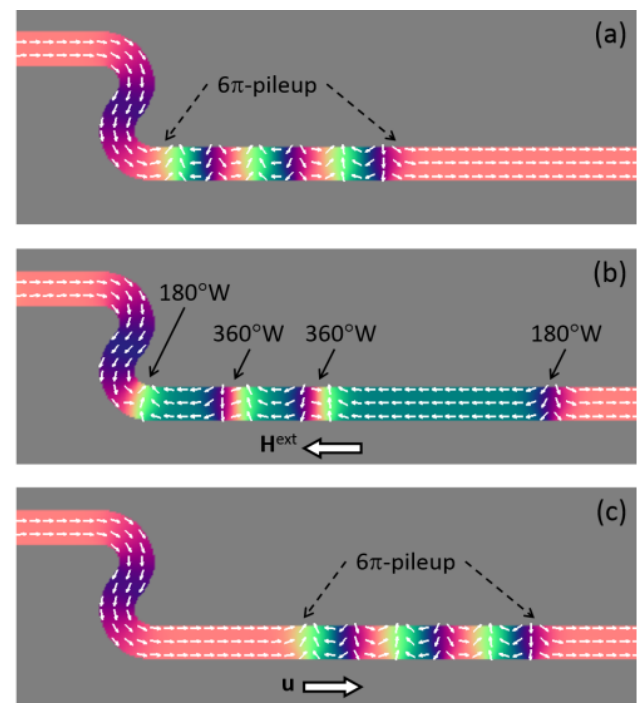

FIG. 1. (Color online) Micromagnetic simulation of domain wall pile-up. (a) A $6 \pi$-pileup near an s-shape bend. (b) Dissociation of $6 \pi$-pileup into two $180^{\circ}$ and two $360^{\circ}$ domain walls under magnetic field. (c) Current-driven motion of $6 \pi$-pileup under spin-polarized current. Small white arrows and color contours within the nanowire represent magnetization direction.

\section{Micromagnetic modeling}

Before discussing the important role of domain wall pile-up in current-driven domain wall depinning in nanowires, we first describe the micromagnetic simulation method used in this study. In micromagnetic modeling, magnetic domain structure is described by the magnetization direction unit vector field $\mathbf{m}(\mathbf{r})$, which is set to zero 
outside the magnetic nanowire. The evolution of the magnetic domain structure driven by spin-polarized current and magnetic field is described by the modified Landau-LifshitzGilbert equation [8]:

$$
\dot{\mathbf{m}}=\gamma \mathbf{H}^{\mathrm{eff}} \times \mathbf{m}+\alpha \mathbf{m} \times \dot{\mathbf{m}}-(\mathbf{u} \cdot \nabla) \mathbf{m}+\beta \mathbf{m} \times[(\mathbf{u} \cdot \nabla) \mathbf{m}],
$$

where $\gamma$ is the gyromagnetic ratio, $\alpha$ the damping parameter, $\beta$ the nonadiabatic spintransfer torque coefficient, $\mathbf{H}^{\text {eff }}$ the effective magnetic field, and $\mathbf{u}$ the electron motion velocity vector. The effective magnetic field is determined by the variational derivative of the free energy with respect to the magnetization vector field (i.e., $\mathbf{H}^{\mathrm{eff}}=d F / d \mathbf{m}$ ), which is a sum of exchange energy, magnetostatic energy, and external magnetic energy [20]:

$$
F=A \int|\operatorname{grad} \mathbf{m}(\mathbf{r})|^{2} d^{3} r+\frac{\mu_{0} M_{s}^{2}}{2} f \frac{d^{3} k}{(2 \pi)^{3}}|\mathbf{n} \cdot \tilde{\mathbf{m}}(\mathbf{k})|^{2}-\mu_{0} M_{s} \int \mathbf{H}^{\mathrm{ex}} \cdot \mathbf{m}(\mathbf{r}) d^{3} r,
$$

where $A$ is the exchange stiffness constant, $\mu_{0}$ the permeability of vacuum, $M_{\mathrm{s}}$ the saturation magnetization, $\mathbf{H}^{\text {ex }}$ the external magnetic field, $f$ the principal value integral excluding the point $\mathbf{k}=\mathbf{0}, \tilde{\mathbf{m}}(\mathbf{k})=\int \mathbf{m}(\mathbf{r}) e^{-i \mathbf{k} \cdot \mathbf{r}} d^{3} r$, and $\mathbf{n}=\mathbf{k} / k$ [21]. The electron motion velocity vector is proportional to the electrical current density $\mathbf{J}$ and determined by $\mathbf{u}(\mathbf{r})=\mathbf{J}(\mathbf{r}) P g \mu_{\mathrm{B}} / 2 e M_{\mathrm{s}}$, where $P$ is the polarization rate of the current and the factor $g \mu_{\mathrm{B}} / 2 e M_{\mathrm{s}}=7 \times 10^{-11} \mathrm{~m}^{3} / \mathrm{C}$ for permalloy [8]. In the simulations, Eq. (1) is numerically solved for a given magnetic field and electric current condition in a magnetic nanowire of given geometry. A $432 \times 432 \times 1$ computational cell with grid size of $4 \mathrm{~nm}$ is used for the patterned thin film of thickness $D=4 \mathrm{~nm}$, and nanowires with width $W=80 \mathrm{~nm}$ are considered in the simulations. The material parameters of permalloy $\mathrm{Ni}_{80} \mathrm{Fe}_{20}$ are used in the simulations: $M_{\mathrm{s}}=860 \mathrm{kA} / \mathrm{m}$ and $A=1.3 \times 10^{-11} \mathrm{~J} / \mathrm{m}$ [17], and $\alpha=0.02$ and $\beta=0.04$ [8].

\section{Results and discussion}
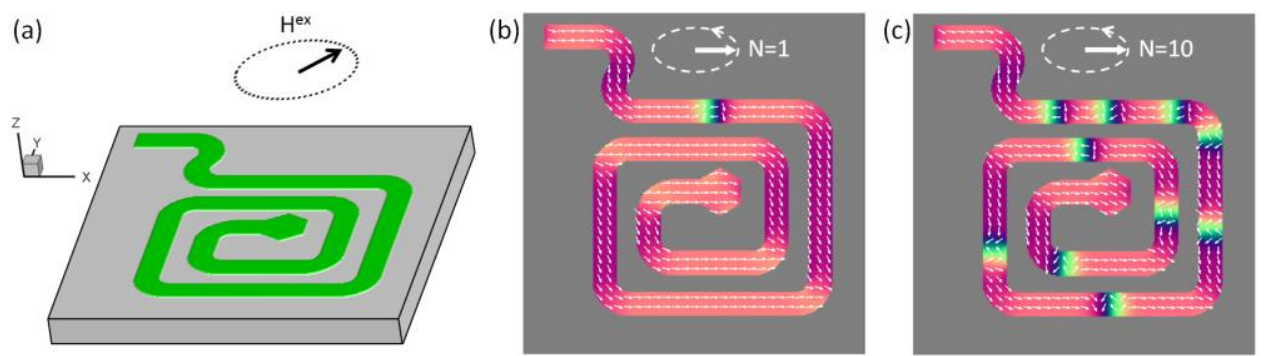

FIG. 2. (Color online) (a) Planar nanowire of patterned magnetic thin film on nonmagnetic substrate. Simulated generation of $360^{\circ}$ domain walls after (b) 1 cycle and (c) 10 cycles of in-plane counterclockwise rotating magnetic field $\mathbf{H}^{\mathrm{ex}}$.

Computer simulation starts from the generation of multiple domain walls of the same chirality to be used for formation of domain wall pile-up. A magnetic nanowire shown in Fig. 2(a) is considered, which combines an s-shape bend as pinning site and a nanowire loop as domain wall generator [20]. Under counterclockwise rotating magnetic field, one $180^{\circ}$ domain wall is generated per half cycle in the alternating sequence of head-to-head and tail-to-tail types. All the generated domain walls possess the same 
chirality (counterclockwise) which is determined by the rotating direction of the applied magnetic field. Under continuously rotating magnetic field, the generated $180^{\circ}$ domain walls propagate along the nanowire loop away from the wall generating element in the center toward the s-shape bend which stops them from propagating further. This process leads to the formation of one $360^{\circ}$ domain wall per full cycle of the rotating magnetic field by conjoining a pair of neighboring $180^{\circ}$ domain walls. The number of $360^{\circ}$ domain walls increases with the number of magnetic field cycles as shown in Fig. 2(b) and (c) respectively for one loading cycle (generating one $360^{\circ}$ domain wall) and ten loading cycles (generating ten $360^{\circ}$ domain walls). Reversing the magnetic field rotating direction reduces the number of domain walls in the nanowire loop by one $360^{\circ}$ domain wall per full cycle of clockwise rotating magnetic field, providing an effective means to control the number of domain walls in the nanowire [20]. The detailed mechanism of domain wall generation, annihilation and storage in such a nanowire design has been studied in our previous work [20]. With a set of domain walls of the same chirality in the nanowire, we consider next the formation of domain wall pile-up at pinning site via current-driven domain wall motion.
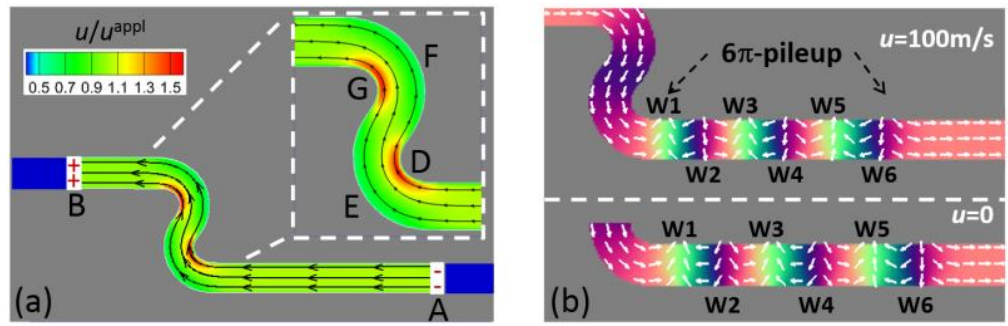

FIG. 3. (Color online) (a) Current crowding in s-shape bend; magnitude and direction of electron motion velocity vector are visualized by color contours and streamlines. (b) $6 \pi$ pileup formed from three separated $360^{\circ}$ domain walls under $u^{\text {appl }}=100 \mathrm{~m} / \mathrm{s}$ (top) and its relaxation at $u^{\mathrm{appl}}=0 \mathrm{~m} / \mathrm{s}$ (bottom); W1-W6 label the six wall elements each providing $180^{\circ}$ magnetization rotation within the pile-up.

Fig. 3(a) shows a section of the nanowire near the s-shape bend where the currentdriven domain wall pinning and depinning behaviors are to be studied. Electrons enter the nanowire at position $\mathrm{A}$ and exit at position $\mathrm{B}$, passing through the s-shape bend. The current density distribution is solved using the phase field method reported previously [22]. The corresponding electron motion velocity vector field is shown by color contours for the magnitude and streamlines for the direction. An inhomogeneous current density distribution develops in a non-straight conducting nanowire. Fig. 3(a) shows the simulated current crowding at the s-shape bend. Current crowding would influence domain wall depinning behavior as will be analyzed later. Under the applied spinpolarized current that yields electron motion velocity $u^{\text {appl }}=100 \mathrm{~m} / \mathrm{s}$ with the local distribution $\mathbf{u}(\mathbf{r}) / u^{\text {appl }}$ shown in Fig. 3(a), Eq. (1) is solved for the magnetic nanowire holding three separated $360^{\circ}$ magnetic domain walls generated by rotating magnetic field as in Fig. 2(c). Hereafter, the current-driven domain wall motions are studied under zero external magnetic field. The simulation results show that all three $360^{\circ}$ magnetic domain walls are driven by current to move toward the s-shape bend then are stopped there (i.e., pinning), and eventually collide into a $6 \pi$-pileup pushing against the s-shape bend as 
shown in the upper part of Fig. 3(b). The individual wall elements each providing $180^{\circ}$ magnetization rotation within the pile-up are labeled W1 to W6 in the order of their positions. When the current is removed, the $6 \pi$-pileup remains but relaxes and becomes wider, as shown in Fig. 1(a) and also in the lower part of Fig. 3(b) for comparison. As aforementioned, the $6 \pi$-pileup dissociates under magnetic field while moves away from the s-shape bend as a whole upon reversal of the current direction, as shown in Figs. 1(b) and 1(c), respectively. To push domain walls through the s-shape bend (i.e., depinning), a higher electric current must be applied, leading to complicated pinning and depinning domain wall behaviors as discussed next.
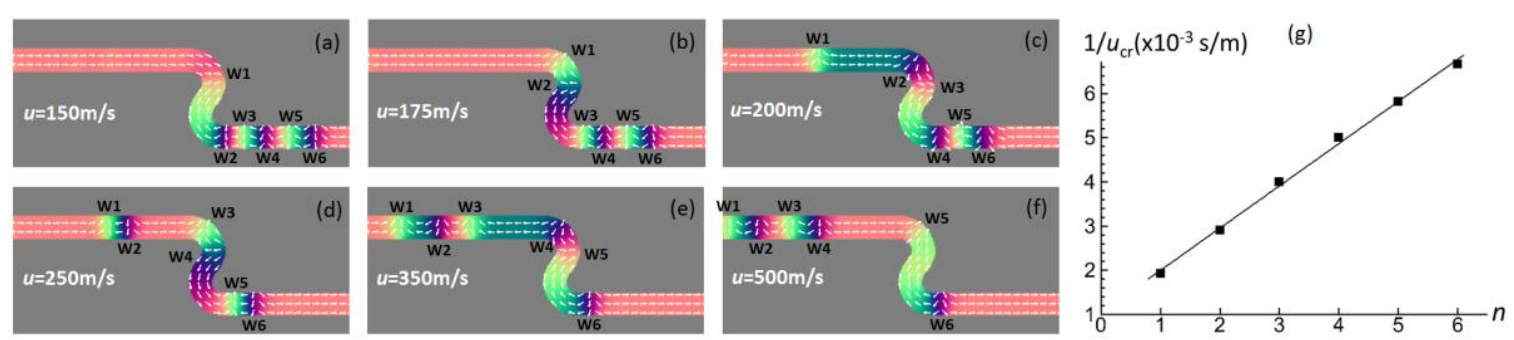

FIG. 4. (Color online) Simulated domain wall pinning and depinning process at the sshape bend under increasing current: (a) $u^{\text {appl }}=150 \mathrm{~m} / \mathrm{s}$, (b) $u^{\text {appl }}=175 \mathrm{~m} / \mathrm{s}$, (c) $u^{\text {appl }}=200 \mathrm{~m} / \mathrm{s}$, (d) $u^{\text {appl }}=250 \mathrm{~m} / \mathrm{s}$, (e) $u^{\text {appl }}=350 \mathrm{~m} / \mathrm{s}$, and (f) $u^{\text {appl }}=500 \mathrm{~m} / \mathrm{s}$. (g) Dependence of the critical depinning current $u_{\mathrm{cr}}$ on the number of domain walls $n$ in the pile-up.

Fig. 4 shows the simulated domain wall pinning and depinning behaviors as the current increases. The s-shape bend is composed of two oppositely curved segments: the lower segment turns clockwise while the upper segment counterclockwise. As will be explained, the lower segment acts as a potential barrier to block domain walls from entering the s-shape bend while the upper segment acts as a potential well that retains domain walls from exiting the bend. At $u^{\text {appl }}=150 \mathrm{~m} / \mathrm{s}$ shown in Fig. 4(a), the first wall (W1) enters the bend leaving behind the rest of walls (W2-W6) in the pile-up; it passes through the lower segment to reach the upper segment and is pinned there. At $u^{\mathrm{appl}}=175 \mathrm{~m} / \mathrm{s}$ in Fig. 4(b), the second wall (W2) follows the same path into the s-shape bend and joins W1. At $u^{\text {appl }}=200 \mathrm{~m} / \mathrm{s}$ in Fig. 4(c), the third wall (W3) follows again the same path into the s-shape bend and joins W2 while W1 is pushed out of the bend into the upper straight segment beyond the s-shape bend. In the same manner, when the current is further increased to $u^{\mathrm{appl}}=250 \mathrm{~m} / \mathrm{s}$ and $350 \mathrm{~m} / \mathrm{s}$ in Fig. $4(\mathrm{~d})$ and (e), the fourth wall (W4) and fifth wall (W5) respectively enter the s-shape bend while W2 and W3 are pushed out of the bend into the upper straight segment. Now three walls (W1, W2, W3) have been transported into the upper straight segment after passing through the s-shape bend, and the rest two walls (W4, W5) are pinned inside the s-shape bend while the last wall (W6) remains blocked from entering the s-shape bend. When the current is further increased to $500 \mathrm{~m} / \mathrm{s}$ in Fig. 4(f), W4 is pushed out of the s-shape bend while W5 is still pinned inside and W6 is still blocked from entering the s-shape bend. This process demonstrates that the critical current for depinning domain walls of an $n \pi$-pileup strongly depends on the number of domain walls, $n$, in the pile-up. For example, depinning wall $\mathrm{W} 1$ to enter the s-shape bend requires $u^{\mathrm{appl}}=150 \mathrm{~m} / \mathrm{s}$ for $n=6$ in Fig. 4(a) while $u^{\text {appl }}=500 \mathrm{~m} / \mathrm{s}$ is not high enough for depinning wall W6 for $n=1$ in Fig. 4(f). To depin a wall to exit the s-shape bend, it requires two walls inside the s-shape bend. For example, 
$u^{\text {appl }}=200 \mathrm{~m} / \mathrm{s}$ can push W1 out when there is another wall behind inside the s-shape bend as shown in Fig. 4(c) while $u^{\text {appl }}=500 \mathrm{~m} / \mathrm{s}$ is not high enough to push wall W5 out when it is the only wall pinned inside the s-shape bend as shown in Fig. 4(f).

To quantitatively determine the dependence of the critical current $\left(u_{\mathrm{cr}}\right)$ on the number of walls $(n)$ in the pile-up, systematic simulations are performed by considering six cases of different domain wall pile-ups with $n=1,2, \ldots, 6$, respectively. Figure $4(\mathrm{~g})$ presents the simulation results of $u_{\mathrm{cr}}$ that is required to depin the first wall of the pile-up to enter the s-shape bend. It is shown that the dependence of the critical current on the number of walls in the pile-up approximately follows an inverse linear relationship of $u_{\text {cr }}$ $\sim 1 / n$ (or $1 / u_{\text {cr }} \sim n$ ). This result can also be understood by analyzing the pinning force to the pile-up and the interaction forces among the individual domain walls in the pile-up, using the force balance equation $(\beta / \lambda) u-f_{\text {pin }}=0$ from the simple one-dimensional domain wall motion model $[9,23]$ where the driving force from the current is balanced by the pinning force $f_{\text {pin }}$ for one static domain wall of thickness $\lambda$. For the case of $n=1$ in Fig. 4(f), W6 experiences the current-induced driving force $(\beta / \lambda) u$ to the left, which is balanced by the pinning force caused by the barrier at the entrance of the s-shape bend. When the current is increased to overcome the barrier of strength $f_{0}$, W6 would be depinned and move into the s-shape bend, and the critical current is $u_{\mathrm{cr}}(n=1)=(\lambda / \beta) f_{0}$ as determined by the force balance equation. For the case of $n=2$ in Fig. 4(d), W6 experiences the current-induced driving force $(\beta / \lambda) u$ to the left and the balancing wallwall interaction force $f_{\mathrm{w} 6}=(\beta / \lambda) u$ to the right exerted by W5; on the other hand, W5 experiences the current-induced driving force $(\beta / \lambda) u$ to the left and the wall-wall interaction force $f_{\mathrm{W} 6}=(\beta / \lambda) u$ to the left exerted by $\mathrm{W} 6$, and their sum is balanced by the pinning force $f_{\text {pin }}$ from the barrier. Therefore, the critical current required to overcome the barrier of strength $f_{0}$ is now $u_{\mathrm{cr}}(n=2)=(\lambda / 2 \beta) f_{0}$, one half of $u_{\mathrm{cr}}(n=1)$. Following the same procedure, it is shown that the critical current for an $n \pi$-pileup is $u_{\mathrm{cr}}(n)=(\lambda / n \beta) f_{0} \sim 1 / n$, in agreement with the simulation results shown in Fig. 4(g). In other words, each individual domain wall in the $n \pi$-pileup contributes to the front domain wall a current-induced driving force of $(\beta / \lambda) u$ through wall-wall interaction force, which effectively reduces the critical current by a factor of $n$. Such a strong dependence of $u_{\mathrm{cr}} \sim 1 / n$ offers an effective means to drastically reduce and tune the depinning current by simply controlling the number of walls in a pile-up.

As the pinning strength of a curved nanowire segment depends on its curvature [6], the curvatures of the lower and upper segments of the s-shape bend can be respectively modified to further tailor its pinning effect. Fig. 5(a) shows the domain wall structures of head-to-head and tail-to-tail $180^{\circ}$ domain walls of counterclockwise chirality in straight nanowire segment, as generated by the rotating magnetic field in Fig. 2. For both types, domain wall width is not uniform across the planar nanowire: wider on the upper edge and narrower on the lower edge, exhibiting an upright bowl shape [24]. Due to such 
asymmetric domain wall structure, curving the nanowire upward and downward would result in different changes in domain wall structure and energy. For example, upward curvature shortens the upper edge and lengthens the lower edge opposing the natural bowl-shaped domain wall, while downward curvature lengthens the upper edge and shortens the lower edge accommodating the natural bowl-shaped domain wall. The former case corresponds to an increased magnetization rotation angle $(\pi+\Delta)$ and increased domain wall energy $(\Delta E>0)$, as shown in Fig. 5(b) compared to Fig. 5(a); the latter case, on the other hand, corresponds to a decreased magnetization rotation angle $(\pi$ $\Delta$ ) and decreased domain wall energy $(\Delta E<0)$, as shown in Fig. 5(c) compared to Fig. 5(a). While Fig. 5(b) and (c) show the effects of nanowire curvature on domain wall structure and energy of head-to-head type of counterclockwise chirality, the same effects are also observed in tail-to-tail type of the same chirality.
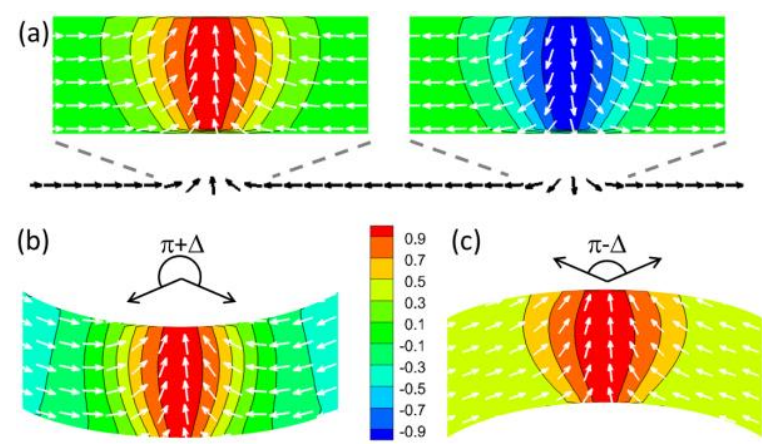

FIG. 5. (Color online) Domain wall structures in a straight nanowire segment (a), and two oppositely curved nanowire segments with (b) increased domain wall width and energy and (c) reduced domain wall width and energy. Small white arrows represent magnetization vectors and color contours represent their components parallel to the domain wall.

Due to an increased domain wall energy $(\Delta E>0)$ shown in Fig. 5(b), the lower segment of the s-shape bend in Fig. 4 acts as a potential barrier [6], blocking domain walls in the lower straight nanowire from entering the bend; and due to a decreased domain wall energy $(\Delta E<0)$ shown in Fig. 5(c), the upper segment of the s-shape bend in Fig. 4 acts as a potential well [6], pinning domain walls inside the bend from exiting into the upper straight nanowire. Therefore, with the lower segment being a potential barrier and the upper segment being a potential well, the s-shape bend serves as pinning element to domain wall motions in the nanowire, as observed in Fig. 4. Domain wall energy depends on both the curvature and the width of the nanowire. When the nanowire width is much smaller than the radius of curvature, the dependence of domain wall energy on the curvature can be described by a simple relationship $|\Delta E| \sim 2 \pi A / R$, where $A$ is the exchange stiffness constant and $R$ the radius of curvature. Correspondingly, the critical depinning current required to overcome such an energy barrier would show a similar proportional dependence on the curvature, $J_{\mathrm{cr}} \sim 1 / R$. When the radius of curvature is comparable to the width of the nanowire, the domain wall energy change and the critical depinning current would deviate from this simple linear dependence on the curvature $1 / R$, which can be evaluated numerically by computer simulations; nevertheless, the critical depinning current would increase when the curvature increases. Figure 6 shows the 
influence of the bend curvature on domain wall pinning strength by considering two sshape bends of different curvatures, $1 / R=1 / 80 \mathrm{~nm}^{-1}$ and $1 / 160 \mathrm{~nm}^{-1}$ ( $R$ being the radius of the nanowire centerline); the former has a higher curvature and is the same as in Fig. 4, while the latter has a smaller curvature thus is bigger in size. The simulation starts with a pinned $360^{\circ}$ domain wall in the lower straight nanowire which consists of two wall elements, W5 and W6, as shown in Fig. 4(d), and $u^{\text {appl }}=400 \mathrm{~m} / \mathrm{s}$ is applied. For the smaller s-shape bend in Fig. 6(a), as expected from the results in Figs. 4(e) and 4(f), the first wall passes through the lower segment to enter the s-shape bend and is subsequently pinned inside by the upper segment, while the second wall remains blocked in the lower straight nanowire. For the larger s-shape bend in Fig. 6(b), both walls pass through the lower and upper segments of the s-shape bend and move into the upper straight nanowire. It shows that the critical depinning current for the larger s-shape bend is lower than $400 \mathrm{~m} / \mathrm{s}$, compared with that higher than $500 \mathrm{~m} / \mathrm{s}$ for the smaller s-shape bend.

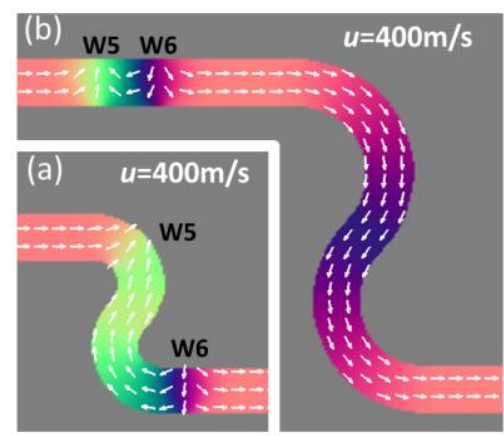

FIG. 6. (Color online) Domain wall behaviors under $u^{\text {appl }}=400 \mathrm{~m} / \mathrm{s}$ at two s-shape bends of different curvatures: (a) $1 / R=1 / 80 \mathrm{~nm}^{-1}$ and (b) $1 / R=1 / 160 \mathrm{~nm}^{-1}$.

Finally, pinning sites (turning corners, bends, joints, and notches) involve certain geometrical shape changes that usually cause current crowding (heterogeneous current density distribution). For example, the current density is high near D and G while low near $\mathrm{E}$ and $\mathrm{F}$ positions in the s-shape bend in Fig. 3(a). Based on Eq. (1), the nonuniformity in current density distribution would influence the current-driven domain wall behaviors. To distinguish the effects of the current density distribution from that of the geometrical shape of the pinning site (e.g., the curvature) on domain wall behaviors, a head-to-head domain wall in a straight nanowire under different current density distribution conditions is simulated. Under given average value of $u^{\mathrm{app}}=300 \mathrm{~m} / \mathrm{s}$, three artificial current density distribution conditions are considered as shown in Fig. 7: case I with uniform current density, case II with higher current density on the lower edge (the narrow side of the domain wall) and case III with higher current density on the upper edge (the wide side of domain wall) that linearly decreases to zero across the nanowire width. The simulation results show that, in all three cases, the domain wall moves to the right as expected, but with different speeds. The domain wall moves at higher speed in case II $(645 \mathrm{~m} / \mathrm{s})$ while at lower speed in case III $(537 \mathrm{~m} / \mathrm{s})$ than in case I $(600 \mathrm{~m} / \mathrm{s})$. Moreover, changes in domain wall structure due to different current density distributions are also observed. Three snapshots of the moving domain walls in the three cases are shown in Fig. 7. The upright bowl-shaped domain wall structure in case I shown in Fig. 7(a) is skewed to the left in case II in Fig. 7(b) while to the right in case III in Fig. 7(c), 
complying with the simple idea that the part of a wall under higher current density is pushed more than the part under lower current density. The observed differences in domain wall speed in the three cases (III, I, II in an increasing order) indicate that the current provides a greater driving force to the wall motion as a whole when the current density is higher at the narrow side than at the wide side of the domain wall. It is because the narrow side possesses a greater magnetization gradient $(\nabla \mathbf{m})$ thus experiences a higher driving force to the current-driven domain wall motion, since the driving force is proportional to the product of the current density and the magnetization gradient as shown in Eq. (1). Therefore, locally distributing a higher current density at the narrow part (with greater magnetization gradient) of the domain wall would increase the driving force to the domain wall motion and thus reduce the critical depinning current as an average current density. Moreover, as different types of domain walls possess different wall structures, the same current density would affect different types of domain walls differently. For example, if the chirality of the domain wall in Fig. 7 is switched to the opposite (clockwise), the domain wall structure changes to upside-down bowl shape and the domain wall speed would be higher in case III than in case II instead. Since the current crowding is always present at a pinning site and the current density distribution is determined by the shape of the pinning site, the effects of the current crowding can be utilized by appropriate design of the pinning site geometry and choice of domain wall chirality.

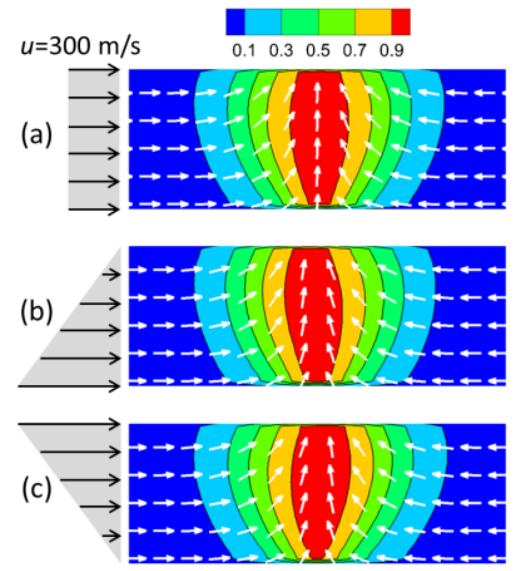

FIG. 7. (Color online) Simulated domain wall structure and motion under (a) uniform, (b) linearly increasing, and (c) linearly decreasing current density across the width of the nanowire.

\section{Conclusions}

In summary, this paper reports an important role of magnetic domain wall pile-up in current-driven domain wall depinning behaviors in magnetic nanowires, which is analogous to the crucial role of dislocation pile-up in determining the mechanical strength of polycrystalline materials (Hall-Petch effect). The critical current for domain wall depinning can be drastically reduced and effectively tuned by simply controlling the number of domain walls in the pile-up. It is shown that domain wall pile-up can be formed by conjoining multiple domain walls of the same chirality, which can be conveniently generated, as exemplified in a magnetic nanowire loop under rotating 
magnetic field. An s-shape bend functions as a pinning site in the simulations, which consists of two oppositely turning curved segments that respectively serve as potential barrier and potential well. The dependence of the pinning strength on the curvature of the bend and the effects of the heterogeneous current density distribution at the pinning site on the domain wall behaviors are discussed.

\section{Acknowledgements}

Support from NSF under Grant No. DMR-1409317 is acknowledged. Simulations were performed on XSEDE supercomputers.

\section{References}

[1] D. A. Allwood, G. Xiong, C. C. Faulkner, D. Atkinson, D. Petit, R. P. Cowburn, Science, 309, 1688 (2005).

[2] S. S. P. Parkin, M. Hayashi, L. Thomas, Science, 320, 190 (2008).

[3] C. H. Marrows, Adv. Phys., 54, 585 (2005).

[4] M. Hayashi, L. Thomas, R. Moriya, C. Rettner, S. S. P. Parkin, Science, 320, 209 (2008).

[5] D. Petit, A. Jausovec, D. Read, R. P. Cowburn, J. Appl. Phys., 103, 114307 (2008).

[6] E.R. Lewis, D. Petit, L. Thevenard, A. V. Jausovec, L. O’Brien, D. E. Read, R. P. Cowburn, Appl. Phys. Lett., 95, 152505 (2009).

[7] A. Yamaguchi, S. Nasu, H. Tanigawa, T. Ono, K. Miyake, K. Mibu, and T. Shinjo, Appl. Phys. Lett., 86, 012511 (2005).

[8] A. Thiaville, Y. Nakatani, J. Miltat, Y. Suzuki, Europhys. Lett., 69, 990 (2005).

[9] J. Ryu, H.W. Lee, J. Appl. Phys., 105, 093929 (2009).

[10] E. Martinez, L. Lopez-Diaz, L. Torres, C. Tristan, O. Alejos, Phys. Rev. B, 75, 174409 (2007).

[11] S.W. Jung, W. Kim, T.D. Lee, K.J. Lee, H.W. Lee, Appl. Phys. Lett., 92, 202508 (2008).

[12] S. Fukami, T. Suzuki, N. Ohshima, K. Nagahara, N. Ishiwata, J. Appl. Phys., 103, $07 \mathrm{E} 718$ (2008).

[13] T. Komine, K. Takahashi, A. Ooba, R. Sugita, J. Appl. Phys., 109, $07 D 503$ (2011).

[14] M.D. Mascaro, C.A. Ross, Phys. Rev. B, 82, 214411 (2010).

[15] S.J. Noh, Y. Miyamoto, M. Okuda, N. Hayashi, Y.K. Kim, J. Appl. Phys., 111, 07D123 (2012).

[16] X. Portier and A. K. Petford-Long, Appl. Phys. Lett., 76, 754 (2000).

[17] M. D. Mascaro, C. Nam, and C. A. Ross, Appl. Phys. Lett., 96, 162501 (2010).

[18] A. Kunz, S. C. Reiff, Appl. Phys. Lett., 94, 192504 (2009).

[19] Y. Jang, S. R. Bowden, M. Mascaro, J. Unguris, C. Ross, Appl. Phys. Lett., 100, 062407 (2012).

[20] L. D. Geng, Y. M. Jin, J. Appl. Phys., 112, 083903 (2012).

[21] A. G. Khachaturyan, Theory of Structural Transformations in Solids (John Wiley \& Sons, New York, 1983).

[22] Y. M. Jin, Appl. Phys. Lett., 103, 021906 (2013).

[23] G. Tatara, T. Takayama, H. Kohno, J. Shibata, Y. Nakatani, H. Fukuyama J. Phys. Soc. Jpn., 75, 064708 (2006). 
[24] H. T. Zeng, D. Petit, L. O’Brien, D. Read, E. R. Lewis, P. R. Cowburn, J. Magn. Magn. Mater., 322, 2010 (2010). 